\title{
Gastrulation in C. elegans ${ }^{*}$
}

Jeremy Nance ${ }^{1, \S}$, Jen-Yi Lee ${ }^{2, \S t}$, Bob Goldstein ${ }^{2, \S}$

${ }^{1}$ Skirball Institute and Department of Cell Biology, New York University
School of Medicine, New York, NY 10016 USA

${ }^{2}$ Department of Biology, University of North Carolina, Chapel Hill, NC 27599 USA

\section{Table of Contents}

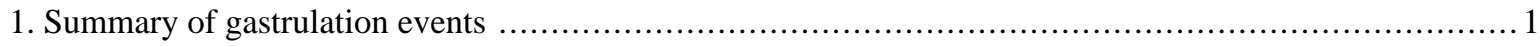

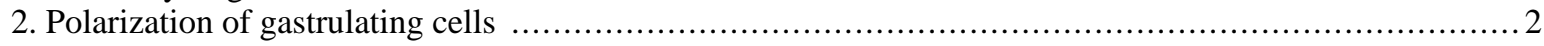

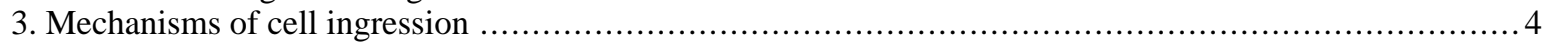

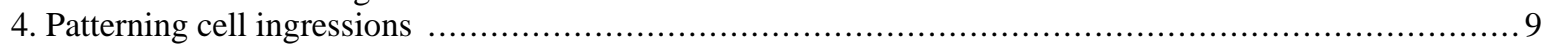

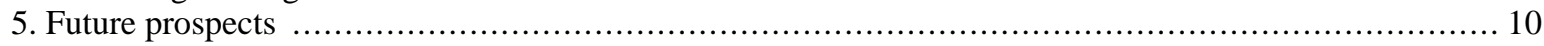

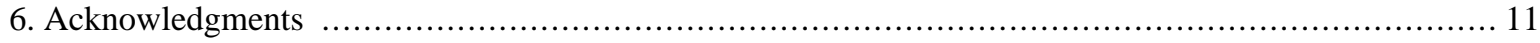

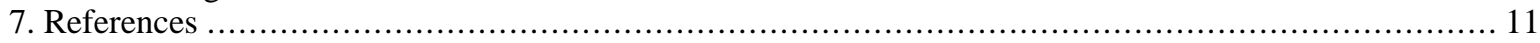

\begin{abstract}
Gastrulation is the process by which the germ layers become positioned in an embryo. C. elegans gastrulation serves as a model for studying the molecular mechanisms of diverse cellular and developmental phenomena, including morphogenesis, cell polarization, cell-cell signaling, actomyosin contraction and cell-cell adhesion. One distinct advantage of studying these phenomena in $C$. elegans is that genetic tools can be combined with high resolution live cell imaging and direct manipulations of the cells involved. Here we review what is known to date about the cellular and molecular mechanisms that function in $C$. elegans gastrulation.
\end{abstract}

\section{Summary of gastrulation events}

Gastrulation in C. elegans is not as overtly dramatic as in many other animal embryos, since cells move only small distances, generally single cell diameters, and the blastocoel space is small. Despite this, gastrulation plays an essential role in development, internalizing endodermal, mesodermal, and germ-line precursors. Gastrulation occurs

\footnotetext{
*Edited by James R. Priess and Geraldine Seydoux. Last revised March 11, 2005. Published September 26, 2005. This chapter should be cited as: Nance, J. et al. Gastrulation in C. elegans (September 26, 2005), WormBook, ed. The C. elegans Research Community, WormBook, doi/10.1895/wormbook.1.23.1, http://www.wormbook.org.

Copyright: (C) 2005 Jeremy Nance, et al. This is an open-access article distributed under the terms of the Creative Commons Attribution License, which permits unrestricted use, distribution, and reproduction in any medium, provided the original author and source are credited.

§ To whom correspondence should be addressed. E-mail: nance@saturn.med.nyu.edu, jenyilee@berkeley.edu or bobg@unc.edu

${ }^{\dagger}$ Current address: Department of Molecular and Cell Biology, University of California, Berkeley, CA 94720 USA
} 
when small groups of cells ingress at various times into the small blastocoel space. The blastocoel space forms when specific surfaces of cells separate from one another in the interior of the embryo. Cells acquire an apical-basal polarity that is important for the asymmetric pattern of adhesions that produce the blastocoel space. Certain PAR proteins adopt apical-basal asymmetries in early embryonic cells and are required to properly pattern cell adhesions.

The endoderm is the first tissue to internalize during gastrulation. Ea and Ep, the endoderm precursor cells, constrict their outer (apical) surfaces as they ingress. Apical constriction may provide a force that draws the neighbors of Ea and Ep towards each other, displacing Ea and Ep into the center of the embryo. Myosin accumulates at the apical surfaces of cells as they ingress, and myosin activity is required for ingression. PAR proteins found on the apical surface are required for the apical accumulation of myosin in ingressing cells and ingressions are inefficient in PAR-depleted embryos.

Cells ingress at reproducible times and positions throughout gastrulation. It is possible that the cellular mechanisms used in Ea and Ep are reused by various other ingressing cells, since myosin also accumulates at the apical surfaces of later ingressing cells. Cell fate appears to play an important role in determining which cells ingress.

Movies of C. elegans gastrulation can be seen at the following web sites:

http://www.bio.unc.edu/faculty/goldstein/lab/movies.html

http://dev.biologists.org/cgi/content/full/130/22/5339/DC1

Variation in gastrulation patterns among other species of nematodes is addressed in another WormBook chapter:

Embryological variation during nematode development.

\section{Polarization of gastrulating cells}

The cell movements of gastrulation begin at the 26-cell stage when the two endodermal precursors, Ea and Ep, move from the surface of the embryo into a small interior cavity called the blastocoel (Sulston et al., 1983). Prior to the 26-cell stage, the embryo is organized as a hull of cells one cell in radius that is surrounded by a vitelline envelope and eggshell. Each cell adopts a reproducible position within the hull and has up to three different membrane surfaces: an outer surface that faces the vitelline envelope (apical surface), surfaces that contact adjacent cells (lateral surfaces), and a surface that faces cells on the opposite side of the hull (basal surface; Figure 1A). The blastocoel forms when basal surfaces of cells separate from one another while lateral surfaces remain adherent (Figure 1C,D; Nance and Priess, 2002). The pattern of cell contacts, rather than adhesive differences between different types of cells, appears to control formation of the blastocoel. For example, a blastocoel-like cavity can form when a single cell (the AB blastomere) is isolated from the embryo and allowed to divide in culture (Figure 1B; Nance and Priess, 2002). This observation suggests that cell contacts induce an apical-basal polarization in cells that causes their different surfaces to develop different adhesive properties.

The localization of certain PAR polarity proteins defines an apical-basal axis in early embryonic cells. PAR-3, a conserved protein with three PDZ domains that is homologous to Drosophila Bazooka, and PAR-2, a RING finger protein, are cortically enriched proteins that are required for anterior-posterior polarity and occupy reciprocal anterior and posterior cortical domains in the zygote and germ-line precursor cells (see WormBook chapter: Asymmetric cell division and axis formation in the embryo). During the four-cell stage, PAR proteins develop a new asymmetry along the apical-basal axis of cells (Etemad-Moghadam et al., 1995; Guo and Kemphues, 1995; Boyd et al., 1996; Hung and Kemphues, 1999; Nance and Priess, 2002; Nance et al., 2003). During the early four-cell stage, PAR-3 is present around the entire cortex of somatic cells in the embryo (Figure 2A). By the end of the four-cell stage, when cell separations that give rise to the blastocoel first become apparent, cortical PAR-3 becomes largely restricted to apical surfaces (Figure 2B). PAR-2 is localized in a reciprocal pattern at the basolateral cortex (Figure $2 \mathrm{C}, \mathrm{D})$. Other PAR proteins that are asymmetrically localized in the zygote and germ-line precursors develop analogous apical-basal asymmetries: PAR-6, which contains a single PDZ domain, and PKC-3, an atypical protein kinase C, are enriched on apical surfaces, and PAR-1, a serine/threonine kinase, is enriched on basolateral surfaces.

PAR-3 and PAR-2 exhibit the same asymmetric distributions when ectopic cell contacts are created by combining blastomeres in culture, suggesting that the pattern of cell contacts establishes apical-basal PAR asymmetry (Nance and Priess, 2002). In the zygote, the asymmetric localization of PAR proteins is achieved in part 
by cortical flows generated by an asymmetric anterior contraction of the cortical cytoskeleton (see WormBook chapter: Asymmetric cell division and axis formation in the embryo). Analogous apically directed movements of the cytoskeleton occur along the apical-basal axis of early embryonic cells and may function to establish the apical-basal asymmetry in PAR distribution (Munro et al., 2004).
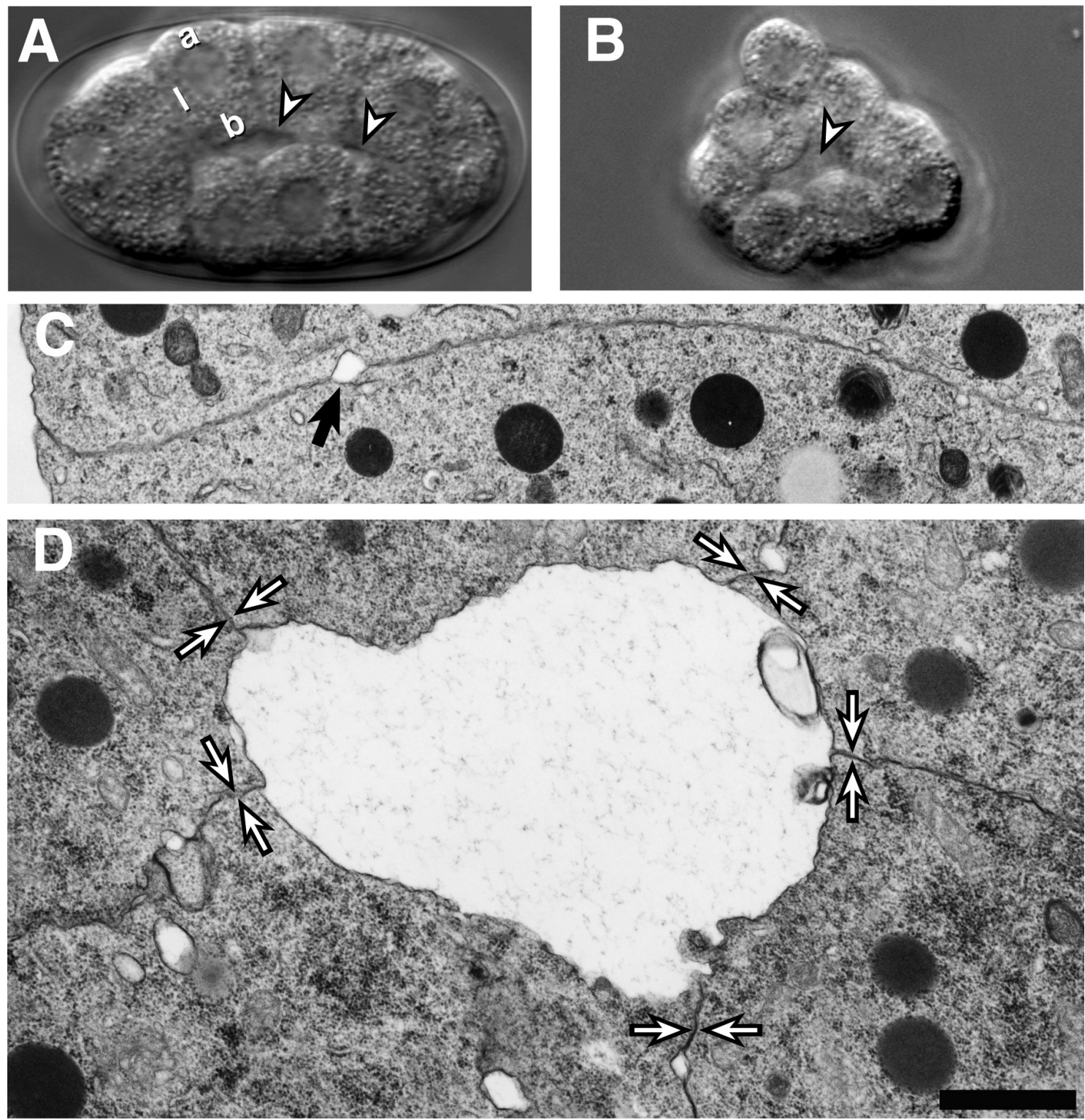

Figure 1. Formation of the blastocoel. Formation of the blastocoel. (A) 26-cell stage embryo. Apical ("a"), lateral ("l") and basal ("b") surfaces of a cell are indicated. The blastocoel (arrowheads) forms between basal surfaces of cells. (B) Descendants of an AB cell that has divided in culture. A blastocoel-like cavity (arrowhead) is present in the center of the cell cluster. (C-D) Transmission electron micrographs of cell contacts in a 28 -cell stage embryo; both panels are shown at the same magnification (bar $=1 \mu \mathrm{m})$. (C) Lateral surfaces of cells are adherent and only occasionally separated by small separations (arrowhead). (D) Large separations can form between the basal surfaces of cells. Cell-cell contacts between lateral surfaces are indicated by opposing arrowheads. Modified and reprinted with permission from Nance and Priess (2002).

PAR-3, but not PAR-2, is required for the asymmetric pattern of cell adhesions observed during blastocoel formation. Cells in par-3 mutant embryos develop spaces between their lateral surfaces similar to those found between basal surfaces in wild-type embryos (Nance and Priess, 2002). To establish that the role of PAR-3 in apical-basal polarity is direct and not a secondary consequence of earlier defects in anterior-posterior polarity, PAR-3 was depleted from early embryonic cells by fusion to a protein domain (the ZF1 domain of PIE-1; Nance et al., 2003). This domain promotes protein degradation beginning at the four-cell stage (Reese et al., 2000; Nance et al., 2003). Embryos expressing only the hybrid PAR-3 protein (par-3(ZF1) embryos) develop separations between the lateral surfaces of cells indistinguishable from those observed in par-3 mutant embryos. Similar phenotypes are 
observed in par-6(ZF1) embryos (Figure 3). The target molecules that mediate the cell adhesion functions of PAR-3 and PAR-6 have yet to be identified.
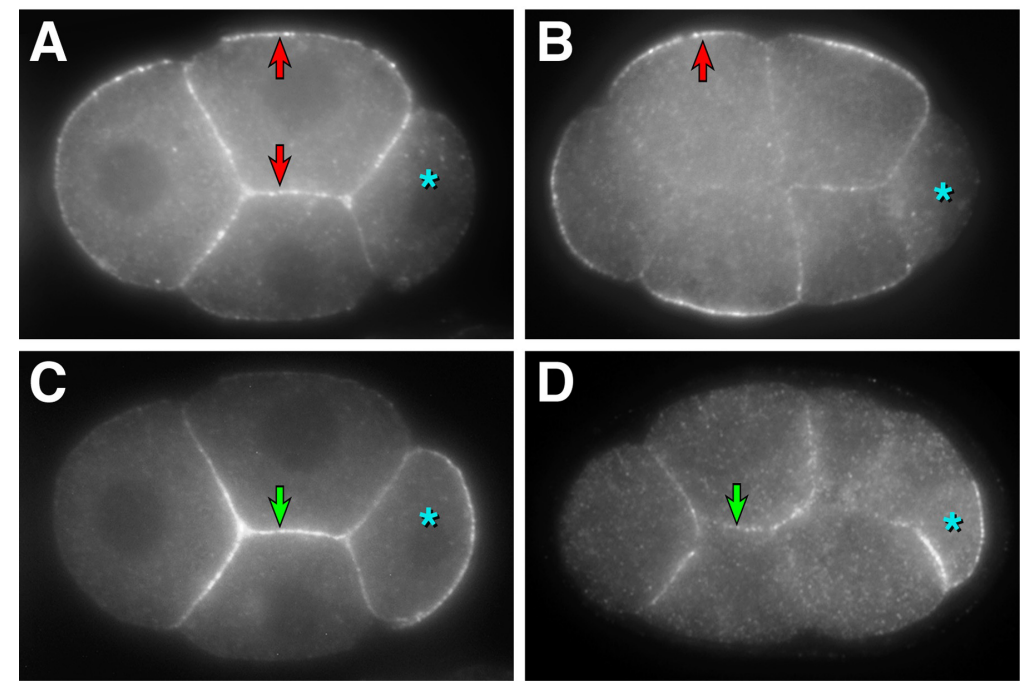

Figure 2. Apical-basal PAR asymmetry. (A, C) Early four-cell stage embryo co-stained with PAR-3 (A) and PAR-2 (C). PAR-3 (red arrows) localizes to the entire cortex of somatic cells; PAR-2 (green arrow) is found at basolateral surfaces of somatic cells. (B, D) 7-8 cell embryos. PAR-3 (B) is enriched at apical surfaces of somatic cells and PAR-2 (D) is found at basolateral surfaces. The germ-line precursor, where PAR proteins exhibit anterior-posterior asymmetries, is indicated with an asterisk in all panels. Modified and reprinted with permission from Nance and Priess (2002).
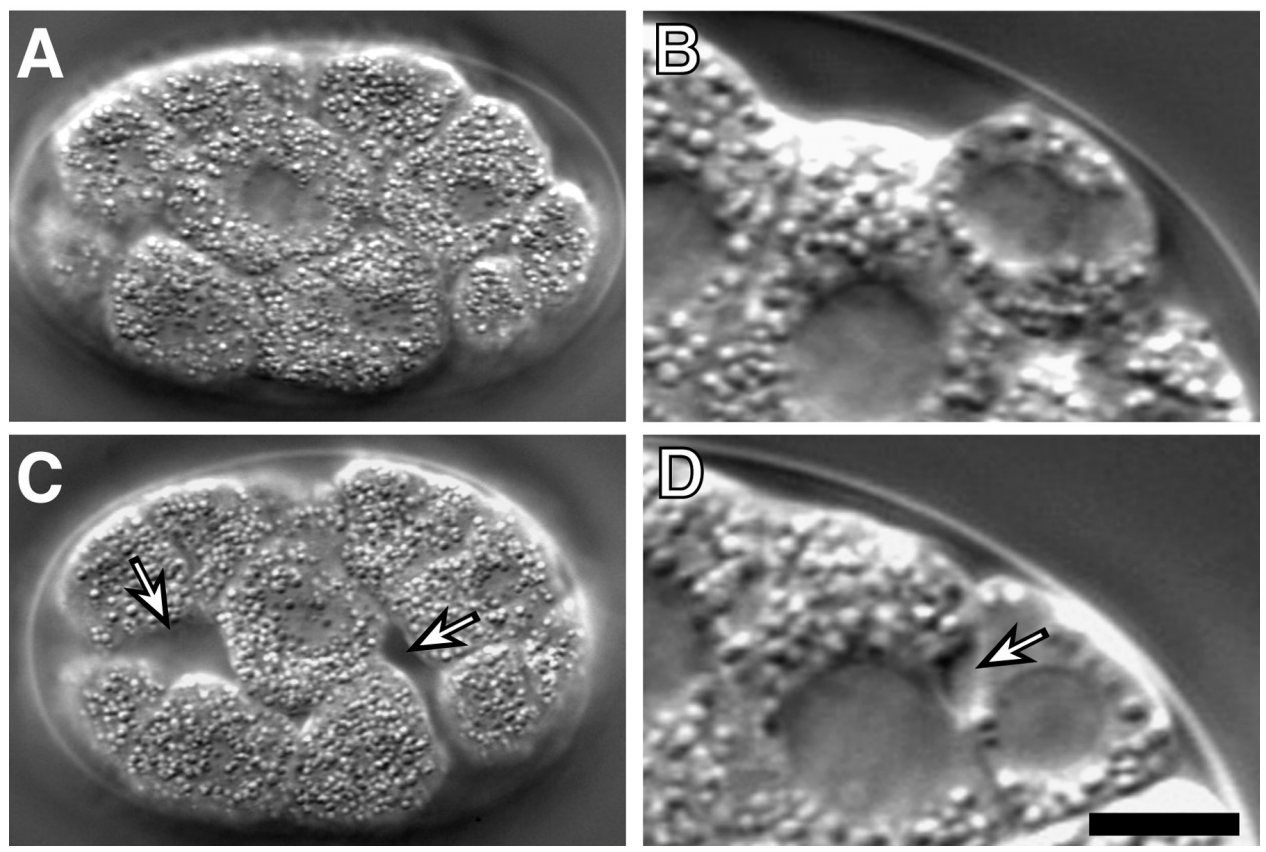

Figure 3. Lateral cell adhesion in $\operatorname{par}(\mathrm{ZF1}$ ) embryos. (A,C) Surface view of a wild-type embryo (A) or an embryo where PAR-6 has been removed by fusion to the ZF1 domain (C, "par-6(ZF1) embryo" ). Spaces (arrows) can be seen between cells in the par-6(ZF1) embryo where lateral cell surfaces do not contact. (B,D) Lateral view of the interior region of a 26-cell stage wild-type embryo (B) or par-6(ZF1) embryo (D). A space (arrow) has formed between lateral cell surfaces in the par-6(ZF1) embryo. Bar $=5 \mu \mathrm{m}$. Modified and reprinted with permission from Nance et al. (2003).

\section{Mechanisms of cell ingression}

As Ea and Ep ingress, their apical surfaces move away from the vitelline envelope and are covered by six neighboring cells: three MS granddaughters (MSap, MSpa, MSpp), two AB progeny (ABplpa, ABplpp) and $\mathrm{P}_{4}$ (Figure 4, Figure 9B; Lee and Goldstein, 2003). In a lateral view, one MS granddaughter (referred to here as MSxx) and $\mathrm{P}_{4}$ can be seen moving toward each other over the apical surfaces of Ea and Ep. To examine whether the 
eggshell and vitelline envelope may provide necessary physical constraints or a necessary microenvironment to the gastrulating embryo, the eggshell and vitelline envelope were removed (Lee and Goldstein, 2003). Ingression movements occur in such devitellinized embryos (Figure 5E-H), indicating that these structures do not provide signals or surfaces essential for ingression.

The descendants of $\mathrm{AB}$ comprise 16 of the 26 embryonic cells present when gastrulation begins, and several $\mathrm{AB}$ descendants contact Ea and Ep (see Figure 9B). To address whether $\mathrm{AB}$ descendants are required for ingression of $\mathrm{Ea}$ and $\mathrm{Ep}$, the $\mathrm{P}$ cell was isolated from the $\mathrm{AB}$ cell at the two cell stage and was allowed to divide in culture (Lee and Goldstein, 2003). Observation of these $\mathrm{P}_{1}$ isolates revealed that MSxx and $\mathrm{P}_{4}$ movements still occur (Figure 5I-P), showing that $\mathrm{AB}$ descendants are not necessary for gastrulation-like movements and ruling out a model in which the complete ring of six neighboring cells is necessary to push the E cells internally.

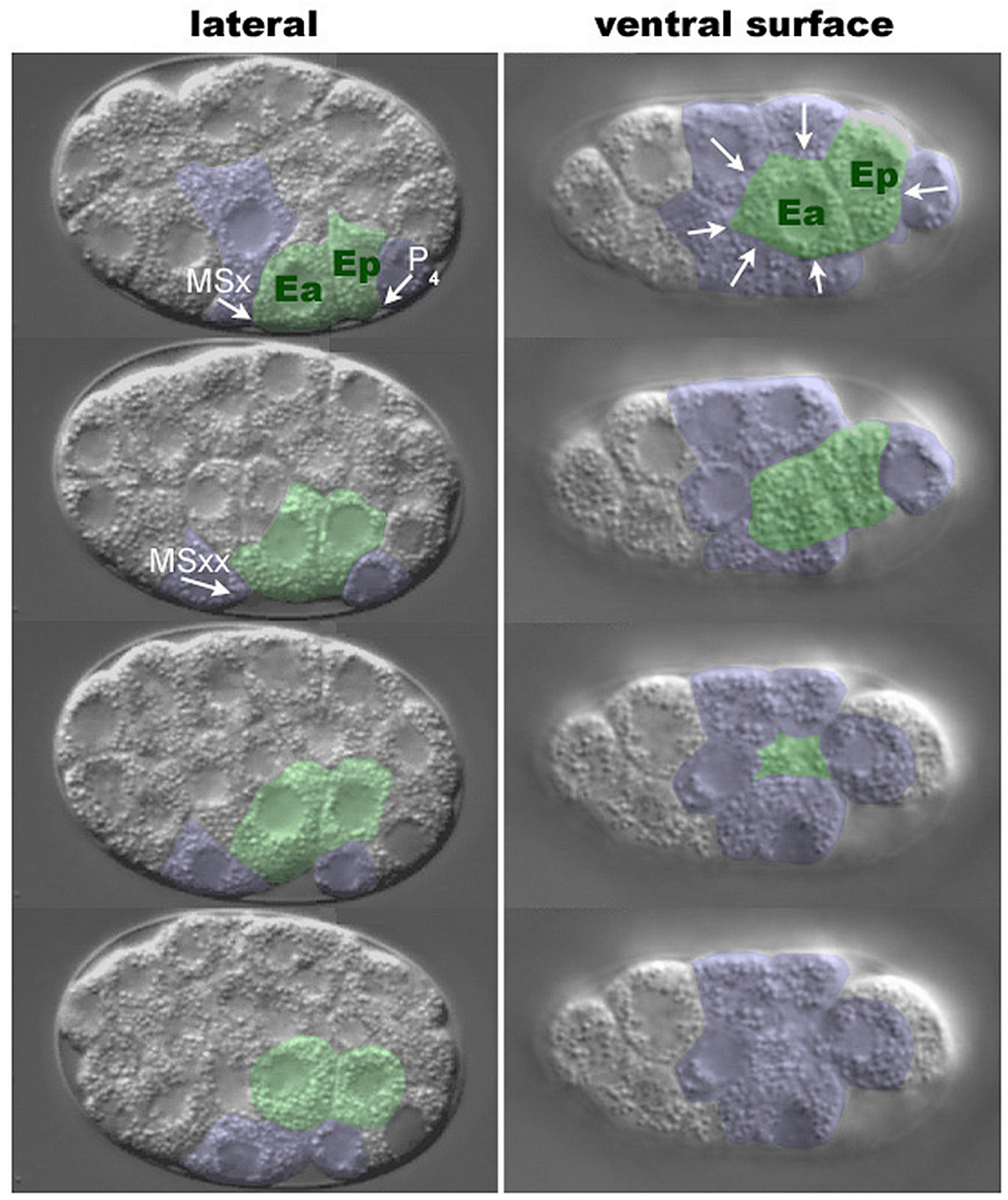

Figure 4. Nomarski time-lapse images of $\boldsymbol{C}$. elegans gastrulation. Ea and Ep are pseudocolored in green, and neighboring cells are in blue. Arrows indicate direction of neighboring cell movement. The left panel shows a lateral view, with the $\mathrm{P}_{4}$ and MSxx labeled (MSx is in the first image, and MSx divides between the first and second images). The right panel shows a ventral view, with Ea and Ep sinking into the embryo. Embryos are oriented anterior to the left. 

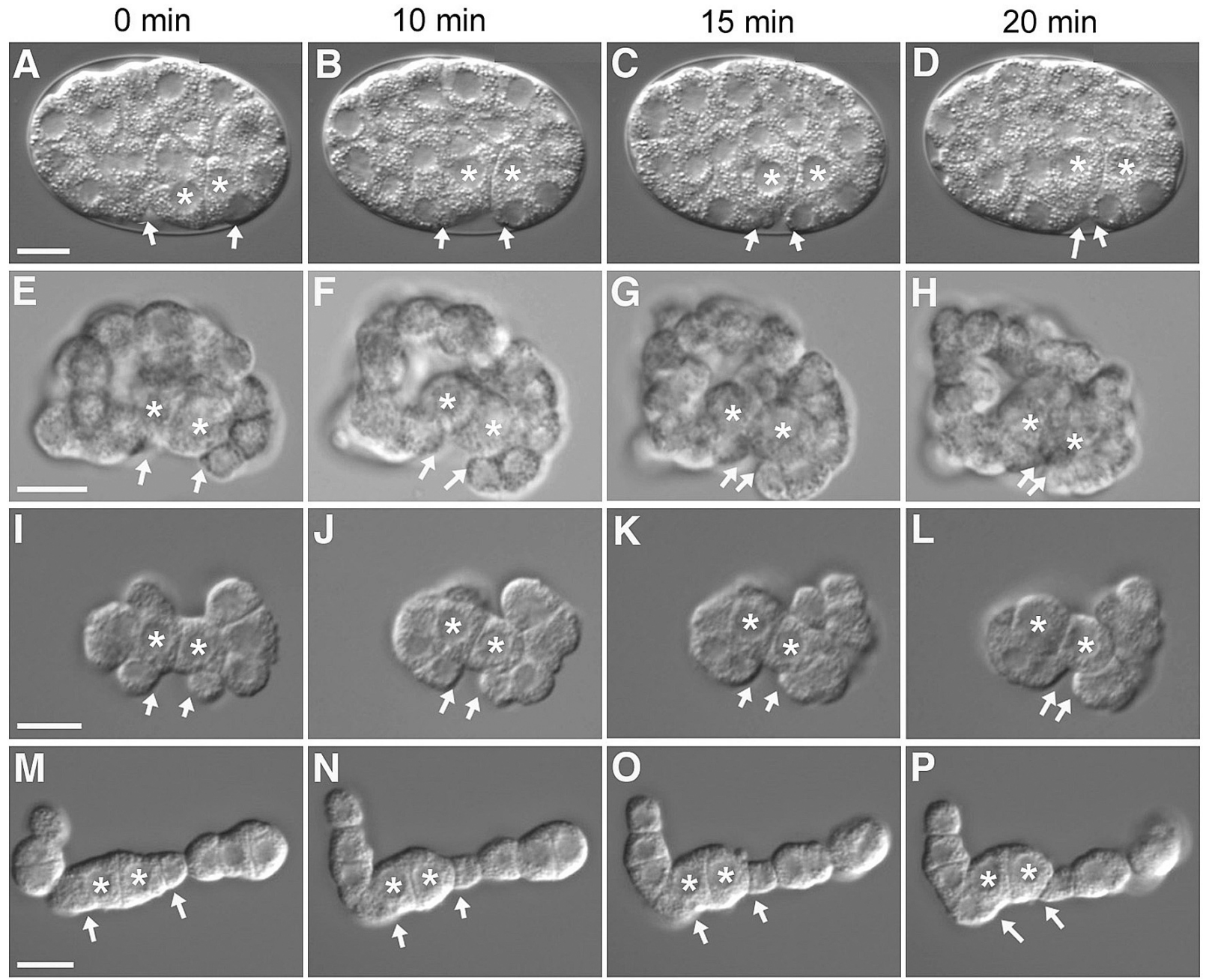

Figure 5. Gastrulation in intact embryos, devitellinized embryos, and $\mathbf{P}$ isolates. Asterisks indicate Ea and Ep, and arrowheads indicate MSxx and $P$ 0 minutes indicates the start of gastrulation movements. (A-D) Time-lapse images of gastrulation in an intact embryo (same embryo as Figure 1C), (E-H) in a devitellinized embryo, and (I-P) in $\mathrm{P}$ isolates. Variation of starting orientation between intact and devitellinized embryos is due to devitellinization (A versus $\mathrm{E}$ ). The two sets of $\mathrm{P}$ isolate images represent two different division patterns, either in a dumbbell orientation (I-L) or in a linear orientation (M-P). Linear orientation occurs in less than $10 \%$ of all $\mathrm{P}$ isolates. Other division patterns were variations between these two extremes. Scale bar $=10 \mu \mathrm{m}$. Reprinted with permission from Lee and Goldstein (2003).

The ingressing endodermal cells control the direction of movement of the neighboring cells (Lee and Goldstein, 2003). $\mathrm{P}$ isolates can divide into a linear array of cells where MSxx cells and $\mathrm{P}$ flank Ea and Ep, respectively (Figure 5M-P). In cases where the $\mathrm{P}_{4}$ cell is removed from a $\mathrm{P}_{\text {isolate, }}$ MSxx can still move toward the Ea/Ep midline, indicating that the $\mathrm{P}_{4}$ cell does not provide a necessary chemoattractant for MSxx movement. $\mathrm{P}_{1}$ isolates can also be separated into two groups and recombined, effectively rotating the cell groups relative to each other (Figure 6). When $\mathrm{P}$ isolates are separated and recombined at the junction of the endodermal cells and either MSxx or $\mathrm{P}_{4}$, MSxx and $\mathrm{P}_{4}^{1}$ move toward one another over the surfaces of Ea/Ep, generally in the same plane, as in unmanipulated $\mathrm{P}$ isolates. However, when Ea and Ep are separated and recombined, MSxx and $\mathrm{P}_{4}$ still move toward the Ea/Ep boundary, but in random axes. Together, these observations show that the polarity of the ingressing endodermal cells determines the direction of movement of neighboring cells, and suggest that neighboring cells may move passively.

Ingressing cells accumulate the non-muscle myosin NMY-2 at their apical surfaces as they ingress. In Drosophila embryos, myosin accumulates at the apical surfaces of cells that undergo a morphogenetic change called apical constriction (Young et al., 1991). Apical constriction is also observed in many other systems, including gastrulating Xenopus bottle cells and ingressing sea urchin primary mesenchyme cells (Keller, 1981; Kimberly and Hardin, 1998). During apical constriction, the width of the apical surface is decreased, altering the shape of the cell. Although not as dramatic as in some other systems, the width of the apical surface also decreases as cells ingress in 
C. elegans. Evidence for apical constriction during ingression comes from experiments where fluorescent microspheres were used to track cell surface movements (Figure 7). Microspheres on the surfaces of Ea and Ep in $P_{1}$ isolates move toward the junction between these cells (Lee and Goldstein, 2003). In addition, non-muscle myosin accumulates at these apical surfaces, and myosin activity is required for ingression (Nance and Priess, 2002; Lee and Goldstein, 2003). These results suggest that an actomyosin-mediated contraction at the apical surface of ingressing cells causes these surfaces to constrict. The ARP- $2 / 3$ complex is also required for ingression, but it is not known whether it plays a role in apical constriction (Severson et al., 2002).

\section{Experimental Schematic}

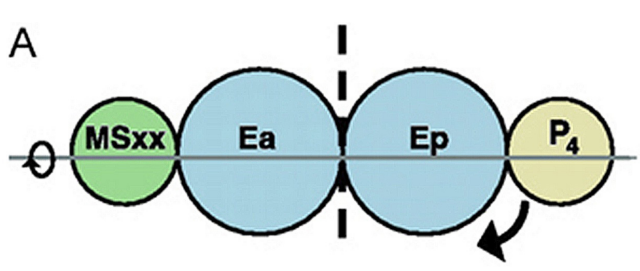

B

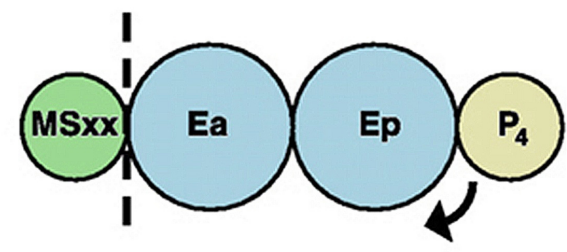

C

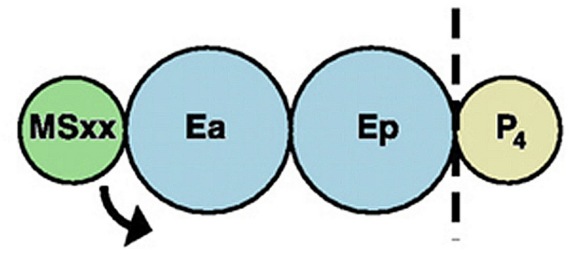

\section{$\underline{\text { Result }}$}
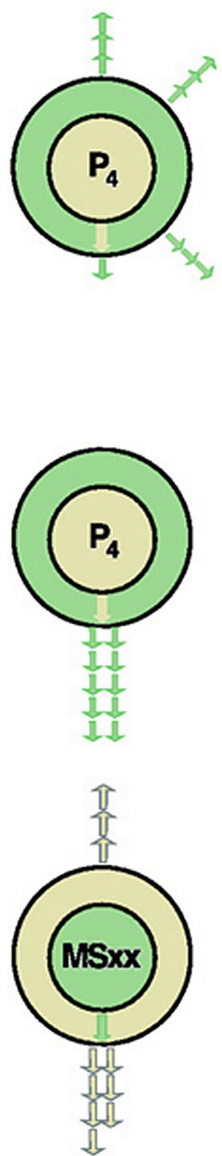

Figure 6. Ea and Ep direct the movement of their neighbors. On the left side of each panel is a schematic drawing of part of a $P$ isolate and an experiment that was performed. Cells in the isolates were separated at the site indicated by the broken line, rotated along the axis indicated by the gray line in A, and then recombined. Black curved arrows indicate the direction of movement of the reference cell. On the right of each panel is a corresponding side view, in which the $\mathrm{P}_{1}$ isolate is oriented as if viewed from one end, either $\mathrm{P}_{4}$ or MSxx (as indicated). Yellow arrows indicate the direction of movement of $\mathrm{P}_{4}$ cells and the green arrows indicate the direction of movement of MSxx cells. (A) Separation and recombination of Ea and Ep, with $\mathrm{P}_{4}$ as the reference cell; (B) MSxx and Ea, with $\mathrm{P}_{4}$ as the reference cell; or (C) $\mathrm{P}_{4}$ and Ep, with MSxx as the reference cell. Modified and reprinted with permission from Lee and Goldstein (2003).

Myosin accumulation in ingressing cells requires PAR-3 and PAR-6. As during blastocoel formation, cortical PAR-3 and PAR-6 are restricted to the apical surfaces of cells during gastrulation. When these proteins are removed from early embryonic cells by tagging with the ZF1 protein degradation domain (see above), cell ingressions proceed much more slowly than in wild-type and the apical surfaces of ingressing cells do not do not visibly accumulate more myosin than neighboring, noningressing cells do (Figure 8; Nance et al., 2003). Thus PAR-3 and PAR-6 function in ingressing cells to promote an apical accumulation of myosin, although it is not yet clear whether the visible apical myosin enrichment is a prerequisite or result of apical constriction. 


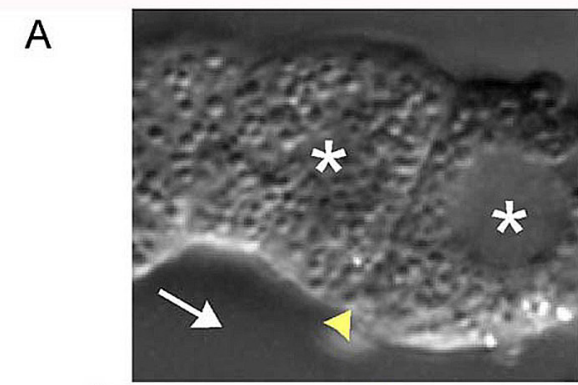

B
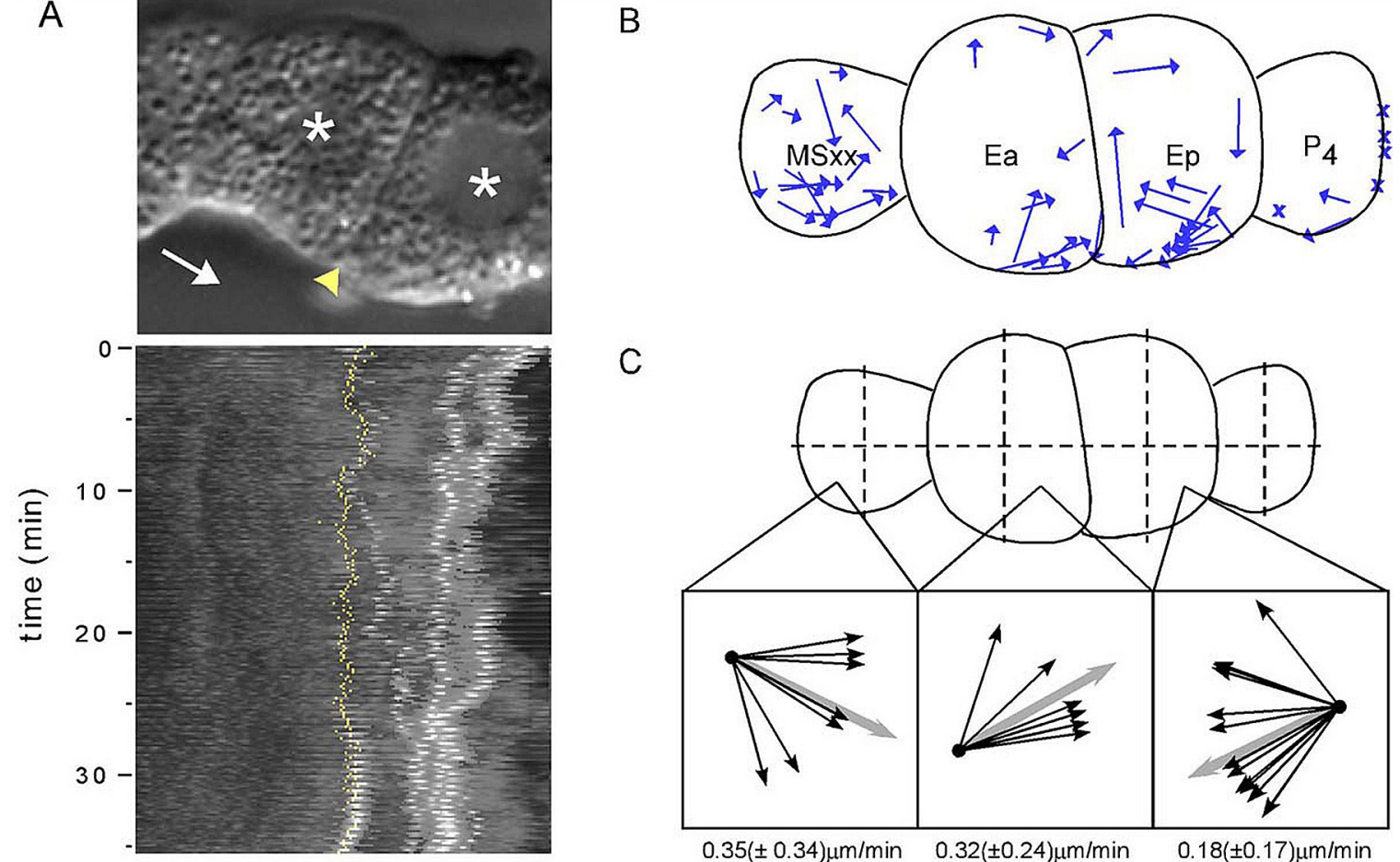

C
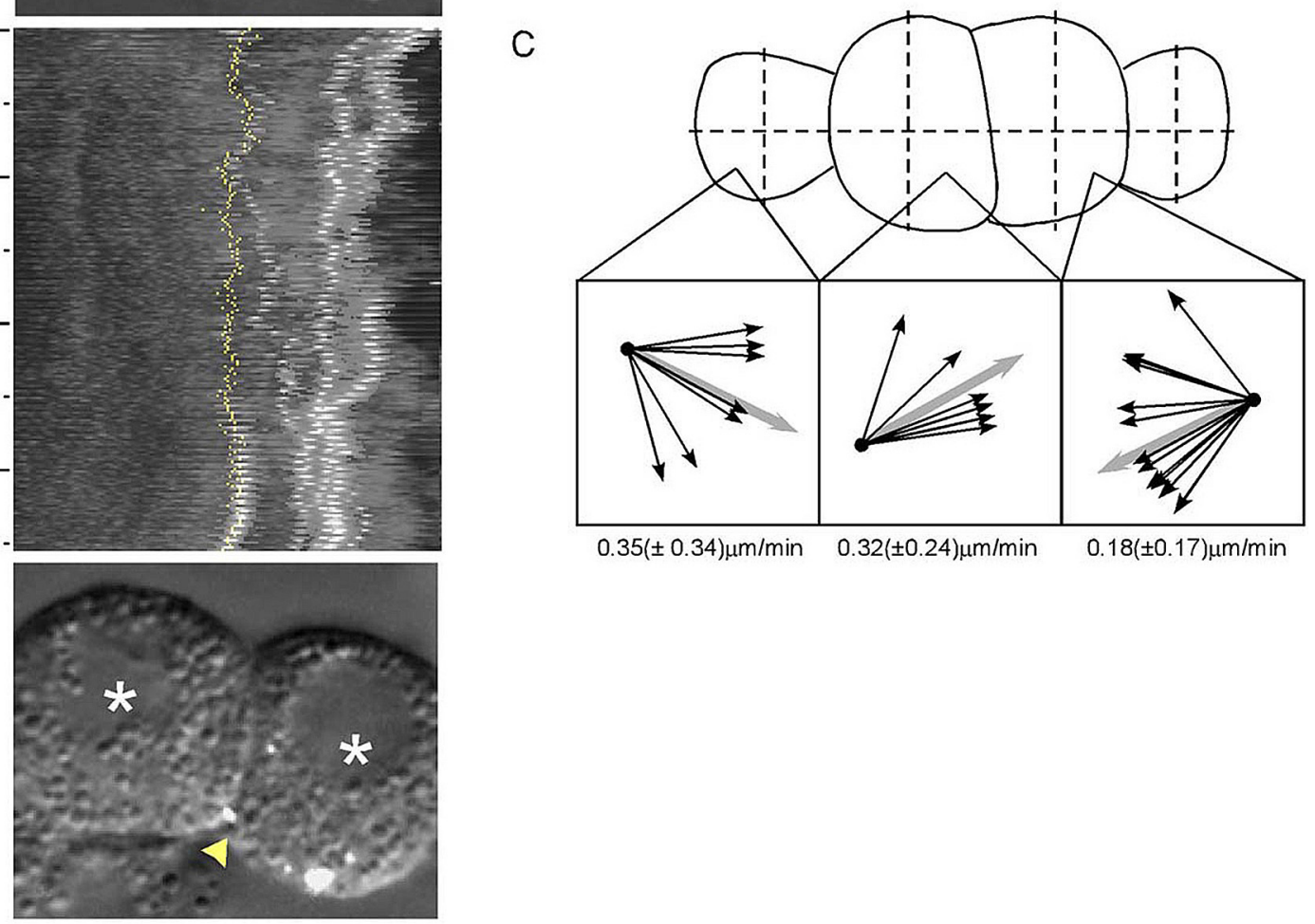

$0.35( \pm 0.34) \mu \mathrm{m} / \mathrm{min} \quad 0.32( \pm 0.24) \mu \mathrm{m} / \mathrm{min}$

$0.18( \pm 0.17) \mu \mathrm{m} / \mathrm{min}$

Figure 7. Apical constriction of Ea and Ep during gastrulation. (A) Kymograph derived from movements of microspheres (white) during gastrulation from one film of a wild-type $\mathrm{P}$ isolate. In the kymograph, the image in each frame of the time-lapse recording is used to generate horizontal lines of image data that are pasted together in descending order; hence time is represented on the y-axis. First and last frame used for the kymograph are above and below the kymograph, respectively. Asterisks label $\mathrm{Ea}$ and $\mathrm{Ep}, \mathrm{Ea} / \mathrm{Ep}$ boundary is marked by yellow arrowhead in still frames and by the yellow line in the kymograph, and the white arrow notes the direction of MSxx movement. The microspheres on Ep can be seen converging toward each other during gastrulation movements. (B) Summary of microsphere movements from 10 films. Each arrow represents the total displacement and angle of movement by each microsphere. " $\mathrm{X}$ " indicates microspheres that did not exhibit any displacement relative to the cell's displacement. (C) Average of the vectors. Insets show the directions of microsphere movement from each quadrant, with the average direction in gray. The average velocity for each set of vectors shown below the box. Reprinted with permission from Lee and Goldstein (2003).

One surprising finding from studies on embryos depleted of PAR-3 or PAR-6 (see above) is that ingression can still occur, albeit slowly, despite the lack of apparent myosin accumulation at the apical cortex (Nance et al., 2003). Whether ingressing cells utilize other mechanims in combination with apical constriction to become internalized is not yet clear. Neither ingressing cells nor spreading neighboring cells appear to use prominent filopodia or lamellipodia to crawl (Lee and Goldstein, 2003). One mechanism that could contribute to ingression is a rolling mechanism of neighboring cells; this is suggested by experiments showing that microspheres coating the apical surface of MSxx cells move toward the endodermal cells (Figure 7; Lee and Goldstein, 2003). This mechanism might be driven by adhesive differences along surfaces of cells, although no adhesion molecules have yet been implicated in gastrulation. 

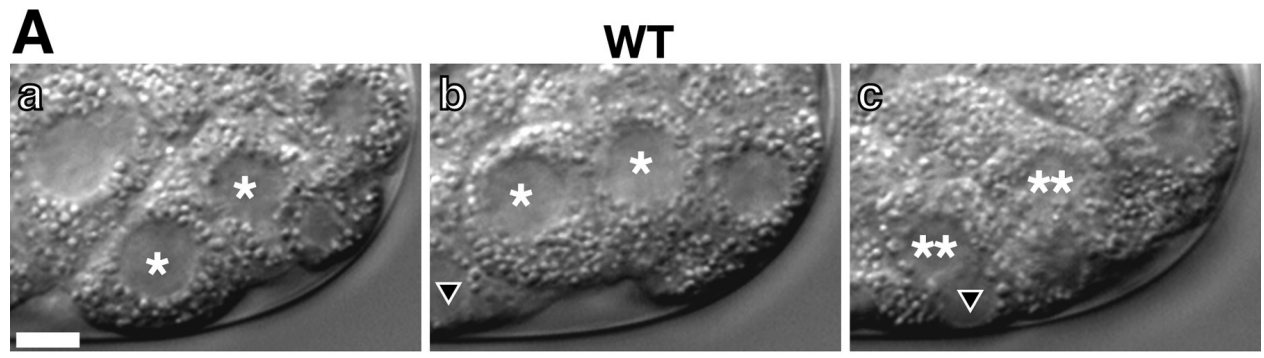

par-3(ZF1)
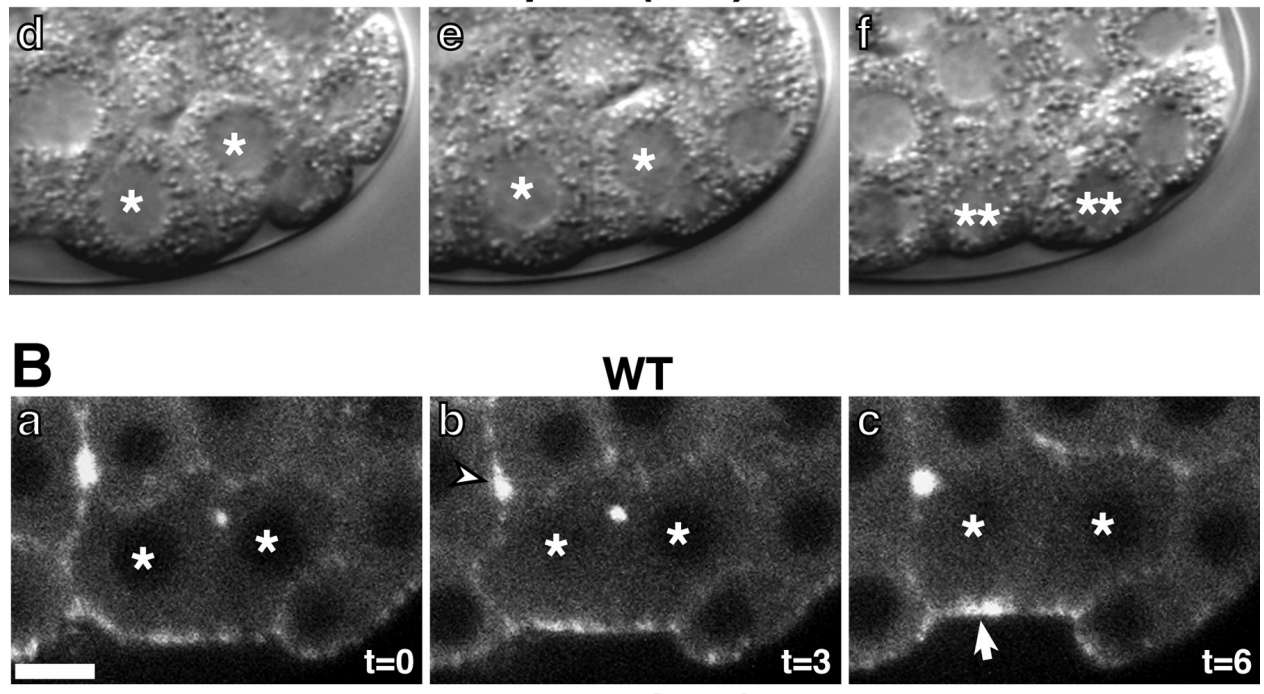

$\operatorname{par-3(ZF1)}$
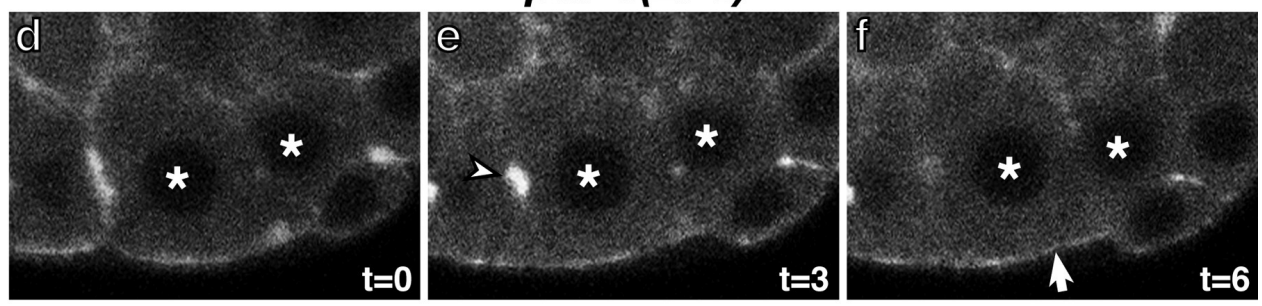

Figure 8. Ingression and apical myosin localization in $\operatorname{par}(\mathbf{Z F 1})$ embryos. (A, a-c) Ea/p (asterisks) position in the same embryo filmed at the 24-cell stage (a), 28-cell stage (b) and 46-cell stage (c). Ea and Ep are internalized and have divided by the 46-cell stage; two of their descendants are indicated with double asterisks. An MSxx cell (black triangle) can be observed moving over the apical surfaces of Ea/Ep. (A, d-f) Ea/p in a par-3(ZF1) embryo at the 24-cell stage (d), 28-cell stage (e) and 46-cell stage (f). Ingression is slow and the descendants of Ea/p (double asterisks) can be seen on the surface of the embryo at the 46-cell stage (A,f). (B) Myosin localization during Ea/p ingression. (a-c) Wild-type embryo expressing myosin-GFP filmed at 3 minute intervals beginning at the 26-cell stage. (d-f) par-3(ZF1) embryo expressing myosin-GFP filmed at 3 minute intervals beginning at the 26-cell stage. Myosin accumulates at the apical surfaces of Ea/p in wild-type (B,c; arrow) but not in par-3(ZF1) embryos $(\mathrm{B}, \mathrm{f}$; arrow). Bar $=5 \mu \mathrm{m}$ Modified and reprinted with permission from Nance et al. (2003).

EMB-5, a putative splicing factor (Schierenberg et al., 1980; Nishiwaki et al., 1993), and GAD-1, a WD repeat protein with some similarity to a transcription initiation factor (Knight and Wood, 1998) are required for ingression, but their roles and the genes that they may regulate have not been defined. Mutations in emb-5 and gad-1 shorten the cell cycle of the endodermal cells, which normally ingress during a single prolonged cell cycle (Schierenberg et al., 1980; Knight and Wood, 1998). Thus EMB-5 and GAD-1 may influence ingression by controlling regulators of the cell cycle.

\section{Patterning cell ingressions}

Ingression of the endodermal cells occurs at approximately 90 minutes after the first cell division of the embryo. After the endodermal cells have completed ingression, mesodermal cells and germ cells ingress from various positions on the ventral surface over the next 200 minutes (Figure 9; Sulston et al., 1983; Nance and Priess, 2002). Although ingressions occur over much of the ventral surface, specific cells ingress at reproducible times and 
positions. A cleavage-arrested endodermal precursor will even gastrulate at the time that its daughters normally would do so (Lahl et al., 2003). These observations suggest that the timing of gastrulation is regulated carefully, and this regulation is distinct to each cell or group of cells.

Several observations suggest that the fate of cells, rather than their ventral position, controls whether they ingress. First, mutations that transform the fates of ingressing cells can prevent the transformed cells from ingressing. For example, genes required for endoderm fate specification are also required for Ea and Ep ingression (ex. skn-1, mom-2, genes in the endoderm determining region; Bowerman et al., 1992; Thorpe et al., 1997; Zhu et al., 1997). Second, transforming the fates of cells can change the time that the transformed cells ingress. Two populations of MS descendants ingress at different times and have different fates: a group of cells called wishbone cells ingresses earlier than another group called central cells (Figure 9C; Sulston et al., 1983; Nance and Priess, 2002). Central cells can be induced to ingress at the same time as wishbone cells if their fates are converted to those of wishbone cells (Nance and Priess, 2002). However, transformed central cells can only be induced to ingress earlier when endodermal cells (which normally lie underneath the central cells) are prevented from ingressing, indicating that cell-cell interactions can also play a role in determining ingression behavior.

After cell ingressions are complete, gastrulation culminates with the epiboly of the hypodermal (epidermal) cells (see WormBook chapter: Epidermal morphogenesis). Hypodermal cells are born on the dorsal surface of the embryo and migrate ventrally to encase the embryo in skin.
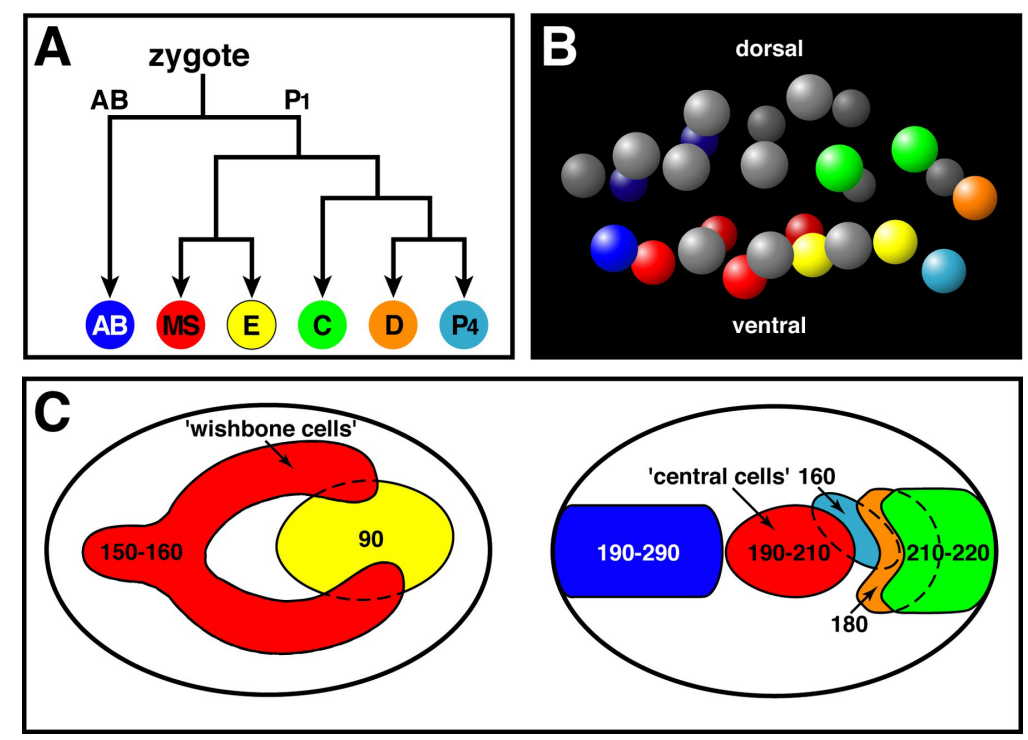

Figure 9. Cell ingressions during gastrulation. (A) Major embryonic cell lineages. Each lineage produces cells that ingress during gastrulation. (B) Position of cell nuclei in a 26-cell stage embryo, lateral perspective, at the onset of gastrulation. Nuclei are colored according to lineage as in (A); AB descendants that do not produce ingressing cells are shown in gray. Note that most ancestors of ingressing cells are born on or near the ventral surface. (C) Map of the ventral surface showing regions of ingression by lineage, colored as in (A); the map is drawn in two panels for clarity. Times of ingression within each region are indicated in minutes after the two-cell stage. Modified and reprinted with permission from Nance and Priess (2002).

\section{Future prospects}

C. elegans gastrulation serves as a model for studying the molecular mechanisms of diverse cell and developmental phenomena (Dawes-Hoang et al., 2003; Putzke and Rothman, 2003). Whereas some of the underlying mechanisms of gastrulation have been resolved (see model in Figure 10), many questions remain. How does apical-basal PAR polarity control apical myosin accumulation in ingressing cells? Does cell adhesion play a role in promoting ingression movements? Does the unique cell cycle length in certain ingressing cells play a role in regulating their ingression? How is ingression triggered and coupled to cell fate? Understanding these issues in this model system may suggest mechanisms by which cells can become repositioned in diverse organisms. 


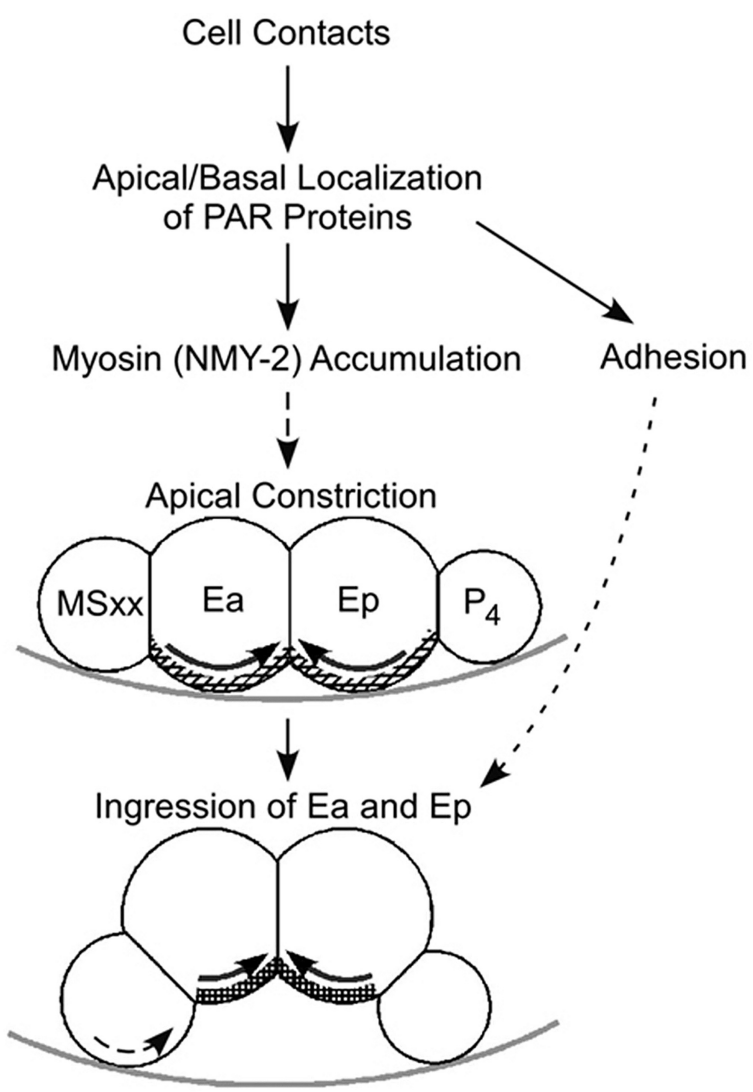

Figure 10. Model for $C$. elegans gastrulation. Cell contacts determine the apical/basal localization of PAR proteins. PAR proteins affect cell adhesion and direct an apical enrichment of myosin. A detectable enrichment may not be required for gastrulation, since gastrulation can occur, although with a delay, in the absence of detectable myosin enrichment. The apical surfaces of Ea and Ep constrict, bringing neighboring cells underneath, between the E cells and the eggshell, and resulting in the ingression of Ea and Ep. Adhesion and MSxx rolling may also play roles in gastrulation movements. Solid arrows denote established relationships or mechanisms, whereas dashed lines denote hypothesized phenomena. Hatched lines on the E cells in the diagram represent the actomyosin network. Light gray line represents part of the eggshell. (modified and reprinted with permission from Lee and Goldstein, 2003 and based on results from Nance and Priess, 2002; Lee and Goldstein, 2003; Nance et al., 2003)

\section{Acknowledgments}

Work on gastrulation by the authors has been supported by Postdoctoral Fellowship Grant PF-02-007-01-DCC from the American Cancer Society (J.N.), funding from the Howard Hughes Medical Institute (J.P.), and a Pew Scholarship in the Biomedical Sciences and NIH R01 grant GM68966 to B.G. (J.-Y.L. and B.G.).

\section{References}

Bowerman, B., Eaton, B.A., and Priess, J.R. (1992). skn-1, a maternally expressed gene required to specify the fate of ventral blastomeres in the early C. elegans embryo. Cell 68, 1061-1075. Abstract Article

Boyd, L., Guo, S., Levitan, D., Stinchcomb, D.T., and Kemphues, K.J. (1996). PAR-2 is asymmetrically distributed and promotes association of $\mathrm{P}$ granules and PAR-1 with the cortex in C. elegans embryos. Development 122 , 3075-3084. Abstract

Dawes-Hoang, R.E., Zallen, J.A., and Wieschaus, E.F. (2003). Bringing classical embryology to $C$. elegans gastrulation. Dev. Cell 4, 6-8. Abstract Article

Etemad-Moghadam, B., Guo, S., and Kemphues, K.J. (1995). Asymmetrically distributed PAR-3 protein contributes to cell polarity and spindle alignment in early C. elegans embryos. Cell 83, 743-752. Abstract Article 
Guo, S., and Kemphues, K.J. (1995). par-1, a gene required for establishing polarity in C. elegans embryos, encodes a putative Ser/Thr kinase that is asymmetrically distributed. Cell 81,611-620. Abstract Article

Hung, T.J., and Kemphues, K.J. (1999). PAR-6 is a conserved PDZ domain-containing protein that colocalizes with PAR-3 in Caenorhabditis elegans embryos. Development 126, 127-135. Abstract

Keller, R.E. (1981). An experimental analysis of the role of bottle cells and the deep marginal zone in gastrulation of Xenopus laevis. J. Exp. Zool. 216, 81-101. Abstract Article

Kimberly, E.L., and Hardin, J. (1998). Bottle cells are required for the initiation of primary invagination in the sea urchin embryo. Dev. Biol. 204, 235-250. Abstract Article

Knight, J.K., and Wood, W.B. (1998). Gastrulation initiation in Caenorhabditis elegans requires the function of gad-1, which encodes a protein with WD repeats. Dev. Biol. 198, 253-265. Abstract

Lahl, V., Halama, C., Schierenberg, E. (2003). Comparative and experimental embryogenesis of Plectidae (Nematoda). Dev. Genes Evol. 213(1), 18-27. Abstract

Lee, J.-Y., and Goldstein, B. (2003). Mechanisms of cell positioning during C. elegans gastrulation. Development 130, 307-320. Abstract Article

Munro, E., Nance, J., and Priess, J.R. (2004). Cortical flows powered by asymmetrical contraction transport PAR proteins to establish and maintain anterior-posterior polarity in the early C. elegans embryo. Dev. Cell 7, 413-424. Abstract Article

Nance, J., Munro, E.M., and Priess, J.R. (2003). C. elegans PAR-3 and PAR-6 are required for apicobasal asymmetries associated with cell adhesion and gastrulation. Development 130,5339-5350. Abstract Article

Nance, J., and Priess, J.R. (2002). Cell polarity and gastrulation in C. elegans. Development 129, 387-397. Abstract

Nishiwaki, K., Sano, T., and Miwa, J. (1993). emb-5, a gene required for the correct timing of gut precursor cell division during gastrulation in Caenorhabditis elegans, encodes a protein similar to the yeast nuclear protein SPT6. Mol. Gen. Genet. 239, 313-322. Article

Powell-Coffman, J.A., Knight, J., and Wood, W.B. (1996). Onset of C. elegans gastrulation is blocked by inhibition of embryonic transcription with an RNA polymerase antisense RNA. Dev. Biol. 178, 472-483. Article

Putzke, A.P., and Rothman, J.H. (2003). Gastrulation: PARtaking of the bottle. Curr. Biol. 13, R223-R225. Abstract Article

Reese, K.J., Dunn, M.A., Waddle, J.A., and Seydoux, G. (2000). Asymmetric segregation of PIE-1 in C. elegans is mediated by two complementary mechanisms that act through separate PIE-1 protein domains. Mol. Cell 6 , 445-455. Abstract Article

Schierenberg, E., Miwa, J., and von Ehrenstein, G. (1980). Cell lineages and developmental defects of temperature-sensitive embryonic arrest mutants in Caenorhabditis elegans. Dev. Biol. 76, 141-159. Abstract Article

Severson, A.F., Baillie, D.L., and Bowerman, B. (2002). A formin homology protein and a profilin are required for cytokinesis and Arp2/3-independent assembly of cortical microfilaments in the early Caenorhabditis elegans embryo. Curr. Biol. 12, 2066-2075. Abstract Article

Sulston, J.E., Schierenberg, E., White, J.G., and Thomson, J.N. (1983). The embryonic cell lineage of the nematode Caenorhabditis elegans. Dev. Biol. 100, 64-119. Abstract Article

Thorpe, C.J., Schlesinger, A., Carter, J.C., and Bowerman, B. (1997). Wnt signaling polarizes an early C. elegans blastomere to distinguish endoderm from mesoderm. Cell 90, 695-705. Abstract Article

Young, P.E., Pesacreata, T.C., and Kiehart, D.P. (1991). Dynamic changes in the distribution of cytoplasmic myosin during Drosophila embryogenesis. Development 111,1-14. Abstract 
Zhu, J., Hill, R.J., Heid, P.J., Fukuyama, M., Sugimoto, A., Priess, J.R., and Rothman, J.H. (1997). end-1 encodes an apparent GATA factor that specifies the endoderm precursor in Caenorhabditis elegans embryos. Genes Dev. 11, 2883-2896. Abstract

All WormBook content, except where otherwise noted, is licensed under a Creative

Commons Attribution License. 